\title{
Telementoring and Teleproctoring in Trauma and Emergency Care
}

\author{
Charles R. Doarn ${ }^{1} \cdot$ Rifat Latifi ${ }^{2}$
}

Published online: 8 July 2016

(C) Springer International Publishing AG (outside the USA) 2016

\begin{abstract}
Purpose of Review The use of telemedicine is long-standing, but only in recent years has it been applied to the specialties of trauma, emergency care, and surgery. We provide a review of its application in these disciplines.

Recent Findings Despite being relatively new, the concept of teletrauma, telepresence, and telesurgery is evolving and is being integrated into modern care of trauma and surgical patients. The role of telementoring for trauma has not been addressed in clinical trials, yet there are consultations and advise on how to manage a critically ill patient, which is telementoring and teleproctoring. Advances in technology, including telemedicine and telepresence applications for trauma, emergency management, and intensive critical care, may be the solution that can reduce, eliminate, or ameliorate the gap in trauma care between rural and urban areas. There is a plethora of examples of the application of telepresence and telemedicine in these fields, although at the current stage, most are only simple examples of the progression of telemedicine in trauma care.

Conclusion As the technology becomes more user friendly and less costly, the hope is that these modalities will become a norm, rather than an exception. What we need is for the trauma community to be creative in finding a sensible way
\end{abstract}

This article is part of the Topical Collection on Telemedicine and Innovative Remote Technologies for Trauma

Charles R. Doarn

charles.doarn@uc.edu

1 Family and Community Medicine, University of Cincinnati, 231 Albert Sabin Way, ML: 0582, Cincinnati, OH 45267-0582, USA

2 Department of Surgery, Westchester Medical Center, Valhalla, NY, USA to help both the patient and physician in the region or hospital where such resources are missing. In this review article, we address a number of elements, including historical notes of telesurgery and other studies pertaining to trauma and emergency care.

Keywords Telemedicine · Telehealth · Telesurgery · Teleproctoring $\cdot$ Telementoring $\cdot$ Teletrauma

\section{Introduction}

Over the past decade or so, healthcare has seen a rapid integration of innovative technologies and approaches, including surgical intervention as well as new tools for expanding the reach of surgeon as skilled provider and proctor or mentor. There are many factors that have enabled this integration. Many of the factors include significant advances in computing power, telecommunications, and imaging acquisition and display. In addition, other non-technical issues have impacted healthcare as well. Trauma and emergency care have benefited from the military's experience over the past two decades of warfare in places like Iraq and Afghanistan. Trauma systems employed today are built on lessons learned from military combat [1]. These lessons not only have led to better protocols and management tools but have also helped establish new and emerging technologies [2]. Robotic surgery is such a tool. The development of these systems has been the direct result of investment by both the Defense Advanced Research Agency (DAPRA) and the National Aeronautics and Space Administration (NASA) in support of their mission needs [3, 4]. In meeting NASA's objective for the U.S. Space Shuttle, International Space Station, and planetary exploration, it has an interest in the development and utilization of robotics. In responding to needs of battlefield medicine, specifically trauma, DARPA was 
interested in developing tools that could be applied in these conditions. These research efforts resulted in a wide variety of technologies with some commercial products that became integrated in civilian healthcare. This innovation has been a key element to the development of telementoring and teleproctoring.

In addition, the ability to monitor patients, where provider and patient are separated by some distance, also known as telemedicine, has greatly improved access to care. The integration of telecommunication and information systems into healthcare provides a lot of portmanteau words or words "fused" together. Telemedicine, telehealth, telesurgery, teletrauma, and telemanipulation are just a few examples. While telemedicine has been an integral part of human exploration of space, it has only recently begun to be an integral part of our daily practice of medicine at all levels [5].

With innovation comes challenges and opportunities. The physician and the surgeon are now trained differently than a generation ago. Today, new tools are used in the pedagogy of a student. Even the World Wide Web has been a significant tool in changing old paradigms of education. If we only consider the surgeon in the "isolated" operating theater, where expertise is present, we may limit our ability to engage expertise from outside that enclosure as well as limit student interaction. Integrating telemedicine in the operating room [6] and developing more intelligent operating suites is only the beginning [7]. The integration of telecommunications and information systems into the operating room, patient exam room, emergency department, a patient's home or the classroom provide new opportunities for telemonitoring and teleproctoring, where knowledge is in a continuous state of growth $[8,9]$.

This paper provides a summary of how telemedicine has been integrated into healthcare, specifically in trauma and emergency medicine in the specific areas of teleproctoring and telementoring.

\section{Currency of Terms}

The ability to manipulate a device from a remote location is called telemanipulation. This can be done synchronously (live or real-time) or asynchronously (store-and-forward) depending on the application. For instance, if a robotic system has been deployed on the surface of Mars, NASA engineers and researchers can send programmable tasks to the system(s) and the system(s) will respond to commands and perform the assigned functions $[10,11]$. Due to delays in communication between Earth and Mars of up to 22 min one way, this form of telemanipulation must be accomplished asynchronously. Performing tasks in real-time or synchronously include a user manipulating an end effector where the control station and controlled system are separated by some distance [12]. This type of function is the basis of surgical robotics like the daVinci system, which is basically a telemanipulation system.
This kind of system can also be used to proctor or mentor someone as well.

As mentioned earlier, U.S. Government investment sparked innovation and development of concepts and eventual commercialization. Computer Motion and Intuitive Surgical developed "telesurgery"-capable robotic "assist" systems, the Zeus and the daVinci, respectively, were innovative systems that came out of this government investment. The concept of "telesurgery" implied the systems could be used where surgeon and robotic system were separated by some distance, either a long cable or communication network.

The concept of telesurgery predicated on providing surgical care where the surgeons and surgical patients were separated by some distance. This could be attributed to both a need and a potential shortage of surgical care capabilities in remote or extreme environments. Such systems could also be used to proctor or guide another surgeon through a training session or even an actual case.

As laparoscopic surgery became the norm for minimally invasive surgery, the telemanipulation systems or surgical "robots" provided the surgeon a unique position, where they were removed from the actual patient by some relatively short distance.

\section{Telemedicine for Trauma}

Live telementoring in the management of a critically ill trauma patient has been proven lifesaving [13]. In a study by Latifi et al., 59 trauma and general surgery patients were evaluated, $35(59 \%)$ were trauma patients, and $24(41 \%)$ were general surgery patients. For 6 of 35 trauma patients, the telementoring via teletrauma consults were considered potentially lifesaving; 17 patients (29\%) were kept in the rural hospitals (8 trauma and 9 general surgery patients).

Case presentation:

An 18-month-old girl was the only survival of a severe car crash with 3 fatalities in Aqua Prieta, Sonora, Mexico. She was transported to Douglas Hospital in southern Arizona in critical condition 3 hours after the crash. She was hypotensive, oxygen saturation was $70 \%$, Glasgow Coma Scale 7, multiple visible injuries to the head, and bilateral lower extremity fractures, and had no intravenous access.

The trauma surgeon in Tucson on call that night received a call from the on duty physician in Douglas, $\mathrm{AZ}$ requesting help and if the patient could be transferred to Trauma Center at the University of Arizona in Tucson. It was her first day on the job, first working day as a physician post family residency, and first day in Arizona! She was scared. A connection was initiated with her through a state-of-the-art telemedicine unit. The trauma surgeon realized why she was visibly 
shaken; she had a little girl dying in her hands. The patient was hypotensive, hypoxic, in a coma, with multiple visible injuries to her head, and fractures of bilateral lower extremities. Forty-five minutes and a few occasionally nerve racking exchanges later, the child was resuscitated and stable enough to be placed on the helicopter for a $150 \mathrm{~km}$ flight to Tucson.

Prior to transfer, the physician in Douglas had intubated the patient successfully, but once she was intubated, the saturation was not coming up as expected. Through the telemedicine link, the trauma surgeon could see the rising of the lower right chest wall with each manual ventilation. The chest radiography clearly demonstrated that the endotracheal tube was in the right lower lobe. Pulling the endotracheal tube back solved the problem of saturation. Grossly dilated stomach was decompressed with a nasogastric tube.

Getting intravenous access in a patient in shock is always difficult, as all the veins are collapsed. The only choice is accessing central veins, such as femoral, jugular, subclavian vein, or osseous access. In a shocked patient, femo$\mathrm{ral}$ access is the fastest and the safest. However, this was this physician's first "femoral stick" in child! Using telemedicine, the trauma surgeon guided the physician in Douglas successfully through femoral line placement.

Once she gained access, the patient could be transfused with packed RBC. Arterial blood gas analysis showed severe acidosis (base deficit 10, from acute blood losses, hemoglobin $5.8 \mathrm{gr} / \mathrm{dL}$ ). After the patient was placed on the helicopter, the joyful, but exhausted and palelooking physician turned to the camera, her face filling the screen and said: "Thank you so much for being with us here today. Without you, this child would have died." The trauma surgeon thanked her and congratulated her and her nursing staff and all others that were involved in saving this child's life, and told her that she had done great and heroic work. This image provides perspective in the true value of telemedicine.

During our first teleresuscitation of a severely injured patient, we learned many things about the teletrauma system and what we needed to have in ours "teletrauma room", such as dosing medication for kids and adults, headphones for a physician and not "a speaker phone", and the angle that we needed to place the camera to see the chest- X- ray. Most importantly, and personally, the trauma surgeon re-confirmed that a calm, deliberate voice with clear directions and clear communication is key in handling major trauma and bad situations. During the 45 minutes, teleresuscitation and the long list of intervention that saved this little girl's life were possible only because the trauma surgeon was able to see what was happening $150 \mathrm{~km}$ away and how the patient was responding to each intervention, almost each second.
The trauma surgeon left the "teletrauma" room, and went to the trauma bay and waited for the patient. The trauma team saved this child only because it had access to advanced technologies. Without the telemedicine technology (the physician in Douglas was right) this patient undoubtedly would have died. No question about that! She was discharged to home after 14 days in the hospital. Her fractures were fixed, ARDS resolved, she was smiling and behaving like any other 18-month-old child. Luckily, her parents were not among the dead in that fatal car crash [14].

Telementoring for trauma and emergency surgery or emergency medicine overall is different than telementoring surgical cases, as we know today. For one, the emergency nature of the injury adds a different dimension. Second, it is rare that the trauma surgeon and the EMS provider, or a physician in a remote healthcare institution that they know each other and work together often enough to be able to coordinate all that is required in saving the life of very sick and or injured patient. ${ }^{1}$

\section{Telemedicine in Surgery}

\section{World's First Telerobotic Surgical Cases}

In September 2002, Professor Jacques Marescaux, who was in New York City, used a Zeus robotic system to perform a cholecystectomy on a patient in Strasbourg, France. This event, known as Operation Lindbergh, was the first true instance of "telesurgery" to be conducted. Marescaux and his team were connected via significant virtual private network (VPN) to the surgical team and patient in France. Using the Zeus "surgeon" unit, he controlled the patient-sided (surgical arms) with end effectors inserted in the patient. This surgical procedure was successful $[11,15]$.

Shortly after this, a Canadian surgeon, Dr. Mehran Anvari, conducted a series of surgical procedures, using a Zeus system between Hamilton and North Bay [16]. Anvari conducted a number of clinical cases during which the surgeon and patient were separated geographically $[17,18]$.

The outcome of both Marescaux and Anvari's work has been reported in the literature and has served as the foundation for most of the research that has followed. Not only can these events lead not only to new and novel surgical capabilities, but they also reinforce the concept of telementoring, where an expert can remotely provide guidance to someone who is located some distance away.

\footnotetext{
${ }^{1}$ This case was reported by the trauma surgeon, Rifat Latifi, MD from the University of Arizona in Tucson and has been reported here with permission of the SJS and the authors
} 
During the mid to late 1990s, NASA funded the development of a commercial space center (CSC) at Yale University under the direction of Dr. Ronald Merrell within the Department of Surgery. Two recognizable and notable surgeons, Dr. Richard Satava and Dr. James "Butch" Rosser, were on staff. The purpose of the CSC, known as the Medical Informatics and Technology Applications (MITA), was to explore and develop test beds in support of NASA's interest in telemedicine both for human spaceflight and its international activities. Merrell and colleagues had become quite adept at providing surgical capability in mobile facilities in the jungles of Ecuador with low bandwidth connectivity to Yale surgeons for mentoring [19]. This concept also proved valuable in mentoring physicians and other providers on Mt. Everest Base Camp [20]. Telementoring is not new or novel, as Dr. Michael DeBakey used the United States' first commercial satellite launched by NASA to mentor a surgical case in 1962 called Early Bird.

Commercial surgical robotic systems were becoming widely available in the early 2000s. The Zeus could support long distance operation and the da Vinci required additional software modifications to permit such operations. The U.S. Army's Telemedicine and Advanced Technology Research Center (TATRC) began to expand its research portfolio in telesurgery. In 2005, TATRC partnered with the University of Cincinnati's Department of Surgery, Johns Hopkins University, Walter Reed Army Medical Center, and Intuitive Surgical to conduct a series of tests with the da Vinci "Classic." The University of Cincinnati (UC) had two systems, one for research and one for clinical needs. The research system was modified to permit remote operations. On two occasions, once from Cincinnati and once from the American Telemedicine Association (ATA) Annual Scientific Meeting in Denver, CO (2005), a nephrectomy was conducted where the porcine model was at Intuitive Surgical's laboratory in Sunnyvale, CA and the surgeon, using the "surgeons" console, was located in Cincinnati and a second time in Denver [21]. This particular series of experiments provided a better understanding of how remote surgery could be performed.

UC and NASA, through TATRC-funded programs, conducted a variety of research efforts where teams of surgeons and astronauts lived in the Aquarius Habitat, a unique underwater laboratory off the coast of Key Largo, FL. This program is known as NASA Extreme Environment Mission Operations (NEEMO) and its infrastructure supported several missions, including NEEMO 7, 9, and 12. On each mission, surgical researchers participated along with several crew members who were physician astronauts and technical personnel either on the surface to evaluate several surgical robotic systems. Components of robotic systems were taken to the habitat, approximately $60 \mathrm{ft}$ underwater and installed so that a series of experiments could be conducted. A Zeus arm was used in
NEEMO 9.SRI provided their M7 and the University of Washington's Bio-robotics Laboratory provided their Raven robotic system for the NEEMO 12 mission. These robotics systems were operated remotely from the ATA Annual Meeting in Nashville (2006) and from the Cincinnati Museum Center to evaluate distance and time delay. These series of activities provided additional opportunity to evaluate and validate the ability to remotely operate a surgical system in an isolated, extreme environment [22].

Additional "telesurgery" experiments were developed and conducted in unique environment to further evaluate the capability of remote surgical capability using SRI's M7 and the University of Washington's BRL's Raven. The Aquarius habitat was wired or tethered to the surface, to provide for the wireless transmission from the water's surface (buoy) to a terrestrial infrastructure. To further evaluate these systems, the concept of telesurgery was to develop a research activity that was supported completely by wireless network. Again, UC partnered with TATRC and UW to deploy its Raven robotic system. An unmanned aerial vehicle (UAV) for communications support was provided by AeroVironment. The research venue was the high desert, north of Simi Valley, CA. A set of experiments were conducted with portable electrical power, a robotic system and a control system, which were separated by a little by a short distance of approximately $100 \mathrm{ft}$. The UAV provided the communications link between the two systems $[23,24]$.

The results of this research have been written up and published in several books and book chapters, including Jacob Rosen, Blake Hannaford, and Richard Satava's book entitled "Surgical Robots;" Sajeesh Kumar and Jacques Marescaux's book entitled "Telesurgery;" and Rifat Latifi's book "Telemedicine for Trauma, Emergencies, and Disaster Management." These are but a few reference tools that provide a foundation for moving telesurgery forward.

In the Rosen book, Moses and Doarn presented a chapter on "Overcoming Barriers to Wider Adoption of Mobile Telerobotic Surgery: Engineering, Clinical and Business Challenges." In this chapter, a number of challenges and barriers were discussed. These lay the foundation for how we might move forward or at least begin a more fruitful discussion.

\section{Paradigms in Teaching_Landscape Changes}

Much of the research on telesurgery has been funded by U.S. military. In Strasbourg, France Dr. Marescaux directs the European Institute of Telesurgery at the Institut de Recherche contre les Cansers de l'Apparell Digestif, where his team continues to develop and refine telesurgical concepts. However, in the USA, funding in the U.S. has diminished. For the concept of telesurgery to become a permanent tool in surgical care, then engineering challenges, non-technical 
challenges, and a strategy must be discussed in a meaningful way and supported with the necessary financial resources to move it forward.

Engineering challenges, many of which have not been adequately addressed, include a variety of thought provoking issues such as technology, access, redundancy, reliability, interoperability, maintenance, ease-of-use, haptics, telecommunications, and latency. Non-technical challenges include (1) personnel; (2) cost of technology, communication, and personnel; (3) cost of inaction; (4) liability; (5) licensing and credentialing; (6) ethics; (7) user acceptance; (8) financial models; and (9) research data; animal trials and human clinical trials.

Perhaps, we are at a crossroads, where telementoring, while not prolific, is an acceptable tool and surgery at a distance is still in development. Telesurgery is not science fiction; it has been shown to work effectively. Although much has been done, there remains a large untouched knowledge base. It has been an interesting journey and with the growing challenges our healthcare system faces, perhaps our journey will get even more interesting.

\section{Conclusions}

For many generations, the surgeon was engaged in his/her practice in a highly controlled, limited access environment. Today, we have integrated telemedicine into the operating room, where it is no longer startling isolated $[6,8,25]$. The ability to bring absent expertise into the trauma bay or operating theater provides opportunities for enabling better outcomes and serves as an excellent pedagogical environment as well. Emergency room protocols have evolved with the integration of new technologies that provide better outcomes and remote surgical proctoring $[26,27]$. Surgical skills are developed over an extensive educational program of residency, fellowship, and ongoing education, including simulations [28]. Some technology today even permits a transference of skills using telerobotic proctoring [29].

With the integration of telemedicine technologies, the ability to respond to trauma has enable better outcomes [30]. The aforementioned case is a testament to the value of telemedicine in trauma. Telemedicine has been shown to be an effective tool in disaster and disaster management as well [31]. As clinicians, we have at our disposal a plethora of non-medical/ surgical tools that can provide us the necessary tools to respond, to train, and to deliver high quality care at other point in time. These tools enable us to reach out virtually and assist our colleagues who may need expertise that is absent at their location or a refresher on a particular intervention. Moreover, telementoring and teleproctoring remove old barriers and provide new avenues for moving medicine and healthcare forward.

\section{Compliance with Ethical Standards}

Conflict of Interest Drs. Doarn and Latifi declare no conflicts of interest relevant to this manuscript.

Human and Animal Rights and Informed Consent This article does not contain any studies with human or animal subjects performed by any of the authors.

\section{References}

1. Chatfield-Ball C, Boyle P, Autier P, van Wees SH, Sullivan R. Lessons learned from the casualties of war: battlefield medicine and its implication for global trauma care. J R Soc Med. 2015;108(3):93-100. doi:10.1177/0141076815570923.

2. Hight RA, Salcedo ES, Martin SP, Cocanour CS, Utter G, Galante JM. Level I academic trauma center integration as a model for sustaining combat surgical skills: the right surgeon in the right place for the right time. J Trauma Acute Care Surg. 2015;78(6):1176-81. doi:10.1097/TA.0000000000000649.

3. Lendvay TS, Hannaford B, Satava RM. Future of robotic surgery. Cancer J. 2013;19(2):109-19. doi:10.1097/PPO.0b013e31828bf822.

4. Takács A, Nagy DA, Rudas IJ, Haidegger T. Origins of surgical robotics: from space to the operating room. Acta Polytech Hung. 2016;13(1):13-30.

5. Doarn CR, Nicogossian AE, Merrell RC. Applications of telemedicine in the United States space program. Telemed J. 1998;4(1):19 30 .

6. Doarn CR. Telemedicine in tomorrow's operating room: a natural fit. Semin Laparosc Surg. 2003;10(3):121-6.

7. Bharathan R, Aggarwal R, Darzi A. Operating room of the future. Best Pract Res Clin Obstet Gynaecol. 2013;27(3):311-22. doi:10.1016/j.bpobgyn.2012.11.003.

8. Merrell RC, Jarrell BE, Schenkman NS, Schoener B, McCullough $\mathrm{K}$. Telemedicine for the operating room of the future. Semin Laparosc Surg. 2003;10(2):91-4.

9. Duftschmid G, Binder M, Wrba T, Dorda W, Pehamberger H. Guidelines for the planning and implementation of telemedical applications. Wien Klin Wochenschr. 2005;117(19-20):673-83.

10. Lii NY, Leidner D, Schiele A, Birkenkampf P, Pleintinger B, Bayer, R. (2015). Command robots from orbit with supervised autonomy: an introduction to the meteron supvis-justin experiment. In Proceedings of the Tenth Annual ACM/IEEE International Conference on Human-Robot Interaction Extended Abstracts (pp. 53-54). ACM.

11. Diana M, Marescaux J. Robotic surgery. Br J Surg. 2015;102(2): e15-28. doi:10.1002/bjs.9711.

12. Modi P, Rodriguez E, Chitwood WR. Robot-assisted cardiac surgery. Interact Cardiovasc Thorac Surg. 2009;9(3):500-5. doi:10.1510/icvts.2009.203182.

13. Latifi R, Hadeed GJ, Rhee P, O’Keeffe T, Friese RS, Wynne JL, et al. Initial experiences and outcomes of telepresence in the management of trauma and emergency surgical patients. Am J Surg. 2009;198(6):905-10. doi:10.1016/j.amjsurg.2009.08.011.

14. Latifi R, Weinstein RS, Porter JM, Ziemba M, Judkins D, Ridings $\mathrm{D}$, et al. Telemedicine and telepresence for trauma and emergency care management. Rev Scand J Surg. 2007;96:281-9.

15. Marescaux J, Leroy J, Gagner M, Rubino F, Mutter D, Vix M, et al. Transatlantic robot-assisted telesurgery. Nature. 2001;413(6854): 379-80. Erratum 2001:414(6865):710.

16. Marescaux J, Rubino F. Telesurgery, telementoring, virtual surgery, and telerobotics. Curr Urol Rep. 2003;4(2):109-13.

17. Anvari M. Robot-assisted remote telepresence surgery. Semin Laparosc Surg. 2004;11(2):123-8. 
18. Anvari M, McKinley C, Stein H. Establishment of the world's first telerobotic remote surgical service: for provision of advanced laparoscopic surgery in a rural community. Ann Surg. 2005;241(3):460-4.

19. Rosser Jr JC, Bell RL, Harnett B, Rodas E, Murayama M, Merrell R. Use of mobile low-bandwith telemedical techniques for extreme telemedicine applications. J Am Coll Surg. 1999;189(4):397-404.

20. Angood PB, Satava R, Doarn C, Merrell R. E3 Group. Telemedicine at the top of the world:the 1998 and 1999 Everest extreme expeditions. Telemed J E Health. 2000;6(3):315-25.

21. Sterbis JR, Hanly EJ, Herman BC, Marohn MR, Broderick TJ, Shih SP, et al. Transcontinental telesurgical nephrectomy using the da Vinci robot in a porcine model. Urology. 2008;71(5):971-3. doi:10.1016/j.urology.2007.11.027.

22. Doarn CR, Anvari M, Low T, Broderick TJ. Evaluation of teleoperated surgical robots in an enclosed undersea environment. Telemed J E Health. 2009;15(4):325-35. doi:10.1089 /tmj.2008.0123.

23. Harnett BM, Doarn CR, Rosen J, Hannaford B, Broderick TJ. Evaluation of unmanned airborne vehicles and mobile robotic telesurgery in an extreme environment. Telemed J E Health. 2008;14(6):539-44. doi:10.1089/tmj.2007.0087.

24. Lum MJ, Rosen J, King H, Friedman DC, Donlin G, Sankaranarayanan G, et al. Telesurgery via Unmanned Aerial Vehicle (UAV) with a field deployable surgical robot. Stud Health Technol Inform. 2007;125:313-5.
25. Raison N, Khan MS, Challacombe B. Telemedicine in surgery: what are the opportunities and hurdles to realising the potential? Curr Urol Rep. 2015;16(7):1-8. doi:10.1007/s11934-015-0522-x.

26. Ereso AQ, Garcia P, Tseng E, Dua MM, Victorino GP, Guy LT. Usability of robotic platforms for remote surgical teleproctoring. Telemed J E Health. 2009;15(5):445-53. doi:10.1089 /tmj.2008.0164.

27. Fleet R, Tounkara FK, Ouimet M, Dupuis G, Poitras J, et al. Portrait of trauma care in Quebec's rural emergency departments and identification of priority intervention needs to improve the quality of care: a study protocol. BMJ. 2016;6(4):e010900. doi:10.1136 /bmjopen-2015-010900.

28. Liss MA, McDougall EM. Robotic surgical simulation. Cancer J. 2013;19(2):124-9.

29. Ereso AQ, Garcia P, Tseng E, Gauger G, Kim H, Dua MM, et al. Live transference of surgical subspecialty skills using telerobotic proctoring to remote general surgeons. J Am Coll Surg. 2010;211(3):400-11. doi:10.1097/PPO.0b013e3182885d79.

30. Latifi R. Telemedicine for Trauma and Intensive Care: Changing the Paradigm of Telepresence. In Technological Advances in Surgery, Trauma and Critical Care. 2015 (pp. 51-57). Springer New York.

31. Rolston DM, Meltzer JS. Telemedicine in the intensive care unit: its role in emergencies and disaster management. Crit Care Clin. 2015;31(2):239-55. doi:10.1016/j.ccc.2014.12.004. 